\section{$\$$ Research Square}

\title{
A study on the biomass wastes' combustion process and their biochar
}

\section{Chaimaa Hadey ( $\nabla$ hadeychaimaa@gmail.com )}

Engineering Sciences and Trades laboratory, ENSAM, University Moulay Ismail, Meknes, Morocco https://orcid.org/0000-0002-6296-9154

\section{Allouch Malika}

Engineering Sciences and Trades laboratory, ENSAM, University Moulay Ismail, Meknes, Morocco

\section{Alami Mohammed}

Engineering Sciences and Trades laboratory, ENSAM, University Moulay Ismail, Meknes, Morocco

\section{Boukhlifi Fatima}

Equip Materials and Applied Catalysis Faculty of Science, University Moulay Ismail, Meknes, Morocco

\section{Research}

Keywords: Biomass, Biochar, Combustion characteristics, kinetic parameters

Posted Date: April 14th, 2020

DOl: https://doi.org/10.21203/rs.3.rs-21123/v1

License: (c) (1) This work is licensed under a Creative Commons Attribution 4.0 International License. Read Full License 


\title{
A Study 0n the biomass wastes 'combustion process and their biochar
}

HADEY Chaimaa ${ }^{1}$, ALLOUCH Malika ${ }^{1}$, ALAMI Mohammed ${ }^{1}$, BOUKHELIFI Fatima ${ }^{2}$

\begin{abstract}
Peanut shells (PS) and sugar canes (SC) constitute an attractive and an energetic biomass source in Morocco since they are renewable, abundant and available. this work seeks to study the thermal decomposition of these biomass samples and their derived solid biofuel under oxidative atmosphere, and it also attempts to determine their kinetic and thermodynamic combustion parameters. The solid biofuel samples were produced by slow pyrolysis at a temperature of $400^{\circ} \mathrm{C}$. Based on the TGA results, the biomass combustion process goes through three stages which are the evaporation of moisture, devolatilization and char formation. For the biochar, it takes two steps that corresponds to the evacuation of water and to the coal combustion. Kinetic parameters of each step are evaluated using Coats-Redfern method, and the thermodynamic parameters are calculated. The results have shown that biochar is less reactive than its original biomass, and that the biomass samples are the most reactive ones in the coal oxidation stage. We have also found that the biomass samples present a different combustion process. These results are useful for the configuration and the design of feasible systems for the conversion of this biomass into energy.
\end{abstract}

Keywords Biomass, Biochar, Combustion characteristics, kinetic parameters

\footnotetext{
$\triangle$ HADEY Chaimaa

hadeychaimaa@gmail.com

1 Engineering Sciences and Trades laboratory, ENSAM, University Moulay Ismail, Meknes, Morocco

2 Equip Materials and Applied Catalysis Faculty of Science, University Moulay Ismail, Meknes, Morocco
} 


\section{Statement of Novelty}

Peanut shells and sugar cane are attractive sources of biomass energy since they are renewable and abundantly available. The new findings of this work are composed of gaining knowledge on kinetic and thermodynamic behavior of biomass decomposition and their biochar in order to evaluate their usefulness in energy and to technically and economically develop feasible systems for the conversion of this biomass to energy.

\section{Introduction}

The growing demand for energy and the depletion of fossil fuels are the main reasons behind the considerable attention that is given to renewable energies [1]. Biomass is considered as an important source of energy, which presents several environmental advantages, thanks to its high abandonment, low price, renewability and carbon-neutrality[2]-[5]. It can also help reduce the emission of toxic gas such as NOx and SOx [6], [7].

Biomass is a difficult fuel to be exploited in its raw state because of several problems that arise such as: a quality diversity, low density and a calorific value. There are several ways to convert biomass into energy such as: the thermochemical and bio-chemical processes [8]. The thermo-chemical conversion technology is dominant because of its high efficiency conversion to gaseous, liquid and solid products under thermal conditions [9][10].

In this context, we are interested in thermochemical conversion and more precisely in slow pyrolysis which aims at maximizing the production of solid biofuel (biochar).

In fact, the biochar, resulting from the slow pyrolysis of biomass is a stable, homogeneous and a clean fuel. Generally, it has both low moisture and fixed carbon contents as well as a heating value that is higher than those of the raw biomass [11]. Biomass chars are found to have porous with highly disordered carbon structure and belong to the class of the most reactive carbon materials. The porosity within the chars causes more accessibility of the reactive gas to active sites resulting in an efficient combustion reactivity [12].

Kinetic and thermodynamic parameters determination seems necessary in order to properly characterize and understand the biomass samples combustion process and their biochar. Thermogravimetric analysis (TGA) is a common technique that is generally used to study the biomass combustion behavior and allow the kinetic parameters estimation, including activation energy (Ea), reaction order and pre-exponential factor using the Coats-Redfern method.

Many researchers have used TGA to study the biomass pyrolysis process [6,8-12] or biomass combustion process [13]-[15]. . However, very few studies of the biomass with its biochar combustion process have been conducted. In this regards, this study aims at providing information on the combustion process of peanut shells, sugar canes and their derived biochar using the TGA technique. The objectives of our works are as follows: (a) the study of biomass and biochar combustion behavior, (b) determination of kinetic model and kinetic combustion parameters of the studied biofuel (c) evaluation of thermodynamic parameters.

\section{Materials and methodology}

\section{Raw materials}

The biomass of this study was the peanut shell and sugar cane produced in the region of Morocco (Fig.1). In the laboratory, the biomass was allowed to dry naturally at room temperature for one week. It was then subjected to oven drying at $105^{\circ} \mathrm{C}$ to constant weight and stored in a desiccator

\section{Solid biofuel Preparation}

The solid biofuel (biochar) is obtained by slow pyrolysis of biomass samples in a muffle furnace under an inert atmosphere. The experiments were carried out at a temperature of $400^{\circ} \mathrm{C}$ for $2 \mathrm{~h}$. (Fig.2) presents the obtained biochar. The biomass and the biochar samples are then crushed on a small scale to have small and more homogeneous samples. Finally, they are stored in a desiccator for ana 
a

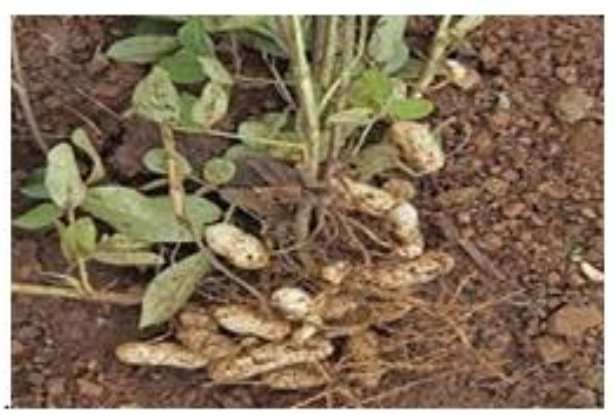

b

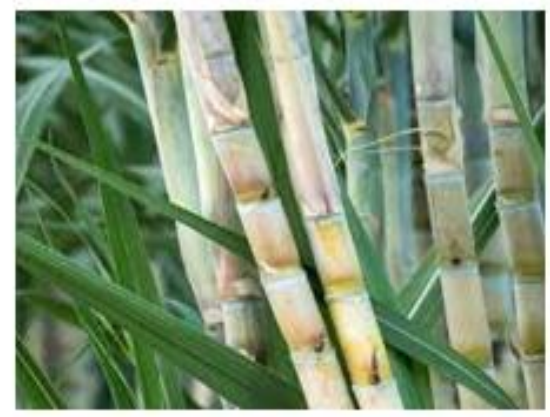

Fig .1 The studied biomass species. a. peanut b. bagasse sugar cane

a

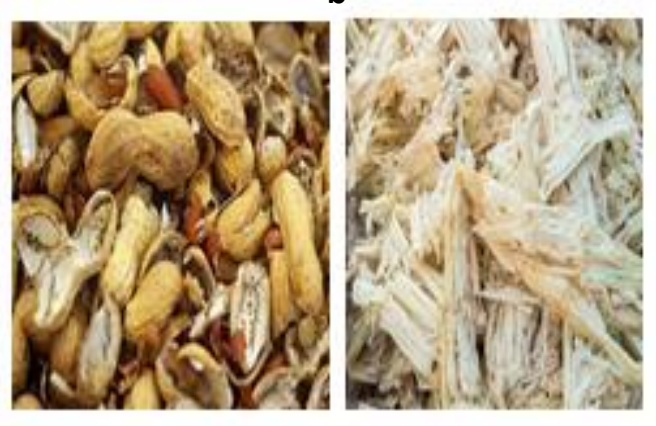

c

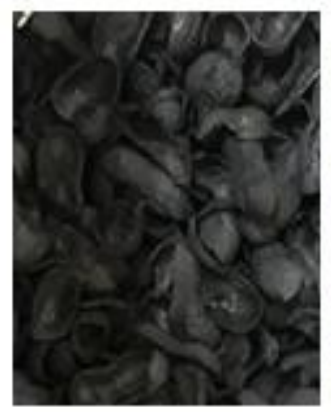

d

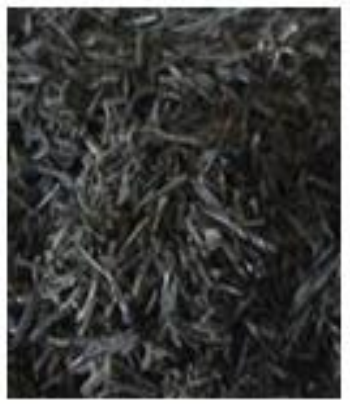

Fig .2 Biomasses and solid biofuel samples. a. Peanut shell (PS) b. sugar cane (PS) c. peanut shell solid biofuel (PS 400$)$ d. sugar cane solid biofuel $\left(\mathrm{PS}_{400}\right)$

\section{Samples' Characterization}

\section{Constitutional and proximate analysis}

Table 1 and 2 show the proximate and the constitutional analysis of the biomasses used in this study. Biomass samples' constitutional analysis is made according to Shiguang method [16]. Proximate analysis measurements are conducted using a thermogravimetric analysis [17]. 
Table 1 Biomass samples' constituent analysis (\%)

\begin{tabular}{ccccc}
\hline Samples & Extractible & Hemicellulose & Cellulose & Lignin \\
\hline PS & 4 & 62 & 22 & 12 \\
SC & 5 & 69 & 16 & 10 \\
\hline
\end{tabular}

Table 2 Biomass samples' proximate analysis (\%)

\begin{tabular}{cccc}
\hline Samples & $\begin{array}{l}\text { Volatile } \\
\text { matters }\end{array}$ & Ash & $\begin{array}{l}\text { Fixed } \\
\text { carbon }\end{array}$ \\
\hline PS & 82.00 & 3.34 & 14.66 \\
SC & 88.01 & 1.99 & 10.00 \\
\hline
\end{tabular}

Infrared spectroscopy

Fourier transform infrared spectroscopy (FTIR) analysis is performed using the FTIR spectrometer to identify the functional groups that are present in the samples. The analysis is carried out by preparing pastilles with $\mathrm{KBr}$ in ratios of 1: 100. The infrared scanning range was 500 to $4000 \mathrm{~cm} \mathrm{-1}$ with a resolution of $4 \mathrm{~cm}-1$.

\section{Thermogravimetric analysis}

The thermal degradation behavior of the biomass samples and their biochar IS determined using a Shimadzu DTG 60 ATG thermogravimetric analyzer. The combustion experiments ARE carried out in air, UNDER a heating rate of $5^{\circ} \mathrm{C} / \mathrm{min}$ from ambient temperature to a temperature of $1000^{\circ} \mathrm{C}$. Slow heating rate IS selected to allow studying the thermal behavior under certain conditions in which the transport processes do not hide the study of the chemistry effects[18].

\section{Kinetic analysis}

The kinetics of decomposition reactions are largely described by Arrhenius's Law which provides information on the reaction rate.

The kinetic equation can be written as follows:

$$
\frac{d \alpha}{d t}=K(T) . f(\alpha)
$$


Where $\alpha=\frac{m_{0}-m}{m_{0}-m_{f}}, m_{0}, m$ and $m_{f}$ are successively the initial mass, the current mass and the final mass. Generally, $\mathrm{K}(\mathrm{T})$ has the form of Arrhenius's law:

$$
K(T)=A \exp \left(-\frac{E a}{R T}\right)
$$

With A: Pre-exponential factor or frequency factor,

E: activation energy,

$\mathrm{R}$ : the universal constant of gases

Since the heating rate $\mathrm{b}$ is constant, it can be expressed by $\beta=\frac{d T}{d t}$.

Thus, combining Eq. (1) and Eq. (2) gives:

$$
\frac{d \alpha}{d T}=\frac{A}{\beta} \exp \left(-\frac{E a}{R T}\right) \cdot f(\alpha)
$$

The $\mathrm{g}(\mathrm{x})$ is the integral function of conversion. By integrating eq.3, we obtain:

$$
g(\alpha)=\int_{0}^{\alpha}, \frac{d \alpha}{f(\alpha)}=\frac{A}{\beta} \int_{T 0}^{T} \exp \left(-\frac{E a}{R T}\right) d T
$$

The expression, $\int_{T 0}^{T} \exp \left(-\frac{E a}{R T}\right) d T$, has no exact analytical solution in that the resolution of the integral is difficult ,and it is only done by numerical solution or using the approximation proposed by Coats and Redfern[9].We obtain :

$\frac{A}{\beta} \int_{T 0}^{T} \exp \left(\frac{-E a}{R T}\right) d T=\frac{A R T^{2}}{\beta E a}\left(1-\frac{2 R T}{E a}\right) \exp \left(\frac{-E a}{R T}\right)$

After dividing T2 and introducing the logarithm, the equation (5) becomes:

$\ln \left(\frac{g(\alpha)}{T^{2}}\right)=\ln \frac{A R}{\beta E a}\left(1-\frac{2 R T}{E a}\right)-\frac{E a}{R T}$

The term 2RTE is much less than 1 for the thermal decomposition of lignocellulosic material [19], so it can be neglected. Eq. 3 can be written in the following form:

$$
\ln \frac{g(\alpha)}{T^{2}}=\ln \frac{A R}{\beta E a}-\frac{E a}{R T}
$$

The activation energy and the pre-exponential factor can be obtained from the slope $\frac{-E a}{R}$ and the ordinate at the origin of the line of the curve $\ln \ln \frac{g(\alpha)}{T^{2}}$ as a function of $\frac{1}{T}$. We HAVE adopted ten kinetic models to study the thermal decomposition of samples (Table 3 ) 
Table 3 Reaction mechanism, model names with their $f(\alpha)$ and $g(\alpha)$

\begin{tabular}{|c|c|c|c|}
\hline Mechanism & Symbols & $\mathrm{F}(\alpha)$ & $\mathrm{G}(\alpha)$ \\
\hline Chemical Reaction-first order [20] & $\mathrm{F} 1$ & $1-\alpha$ & $-\ln (1-\alpha)$ \\
\hline Chemical Reaction -second order & $\mathrm{F} 2$ & $(1-\alpha) 2$ & $(1-\alpha)-1-1$ \\
\hline Chemical Reaction -third order & F3 & $(1-\alpha) 3$ & $((1-\alpha)-2-1) / 2$ \\
\hline Diffusion One Way transport[19] & D1 & $1 / 2 \alpha$ & $\alpha 2$ \\
\hline Diffusion Two Way transport & D2 & $(-\ln (1-\alpha))-1$ & $\alpha+(1-\alpha) \ln (1-\alpha)$ \\
\hline Diffusion Tree Way transport & D3 & $(2 / 3(1-\alpha) 2 / 3 / 1-(1-\alpha) 1 / 3$ & $(1-(1-\alpha) 1 / 3) 2$ \\
\hline Ginstling, Brounshtein equation & D4 & $(2 / 3)(1-\alpha) 1 / 3 / 1-(1-\alpha) 1 / 3$ & $1-2 \alpha / 3-(1-\alpha) 2 / 3$ \\
\hline Zhuravlev, Lesokhin, Tempelman equation & D5 & $(2 / 3)(1-\alpha) 5 / 3 / 1-(1-\alpha) 1 / 3$ & $((1-\alpha)-1 / 3-1) 2$ \\
\hline $\begin{array}{l}\text { Phase Boundary Controlled[19] } \\
\text { R2 - contracting cylinder }\end{array}$ & $\mathrm{R} 2$ & $2(1-\alpha) 1 / 2$ & $1-(1-\alpha) 1 / 3$ \\
\hline Phase Boundary Controlled & R3 & $3(1-\alpha) 2 / 3$ & $1(1-\alpha) 2 / 3$ \\
\hline
\end{tabular}

\section{Thermodynamic parameters Calculation}

Thermodynamic data determination is also important in defining the process feasibility and in performing the energy calculations [21]. From the thermogravimetric analysis, we obtain the thermodynamic parameters such as the change of enthalpy H, entropy S, and the Gibbs free energy variation G. These parameters were calculated by the following equations[22]:

$$
\begin{gathered}
\Delta H=E a-R T \\
\Delta G=E a+R T \ln \frac{K_{B} T}{h A} \\
\Delta S=\frac{\Delta H-\Delta G}{T m}
\end{gathered}
$$


$\mathrm{KB}$ and $\mathrm{h}$ are respectively the Boltzman and Planck constant

\section{Results and discussion}

\section{Infrared spectroscopy}

Fourier transform infrared spectroscopy (FTIR) is used to study the functional groups present in any substance. (Fig.3) shows the spectra with very tense and varied peaks of biomass and biochar samples. We HAVE found that all biomass samples have similar aromatic and aliphatic functional groups that come from the three main constituents of the studied wastes namely cellulose, hemicellulose and lignin. Indeed, these three components are mainly composed of alkenes, esters, ketones, aromatic rings and Alcohol with different functional groups containing oxygen such as $\mathrm{O}-\mathrm{H}(3400-$ $3200 \mathrm{~cm}-1) \mathrm{C}=\mathrm{O}(1765-1715 \mathrm{~cm}-1), \mathrm{C}-\mathrm{O}-\mathrm{C}(1270 \mathrm{~cm}-1)$, and C-O-H $(1050 \mathrm{~cm}-1)[18,[23]$.

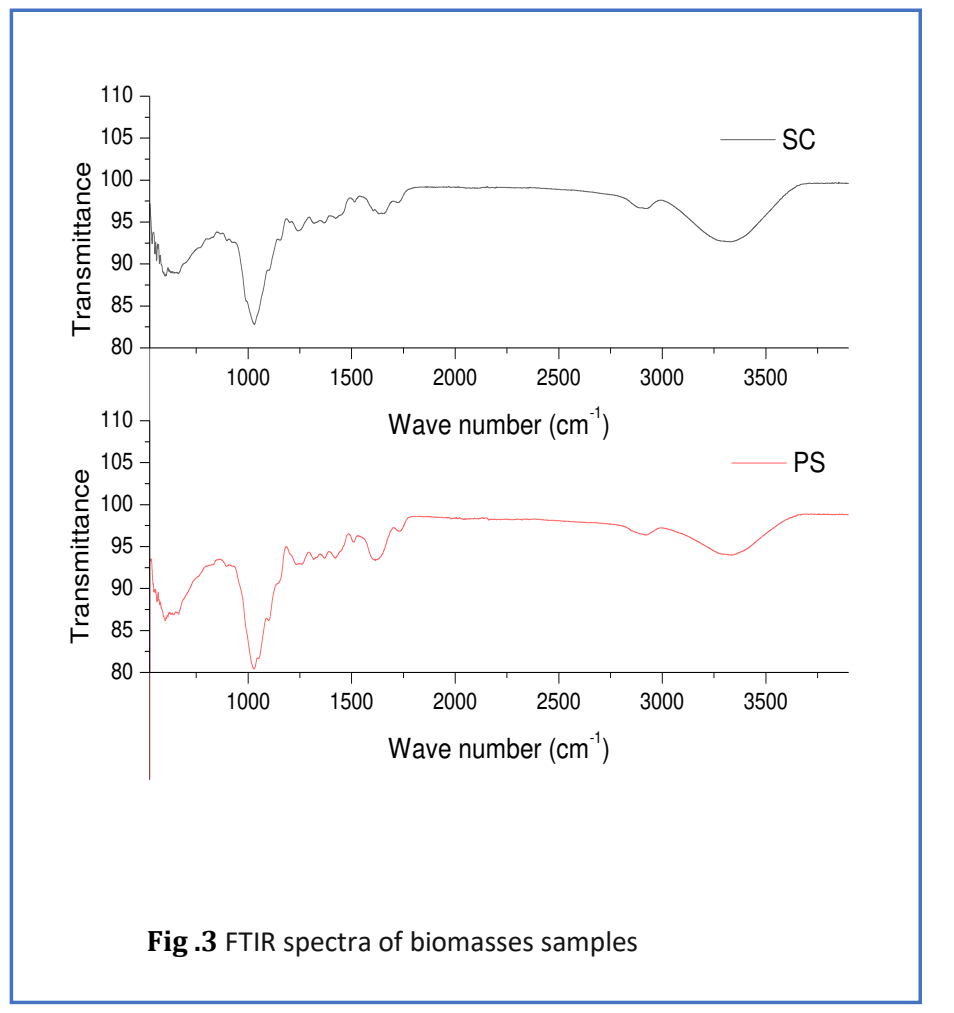




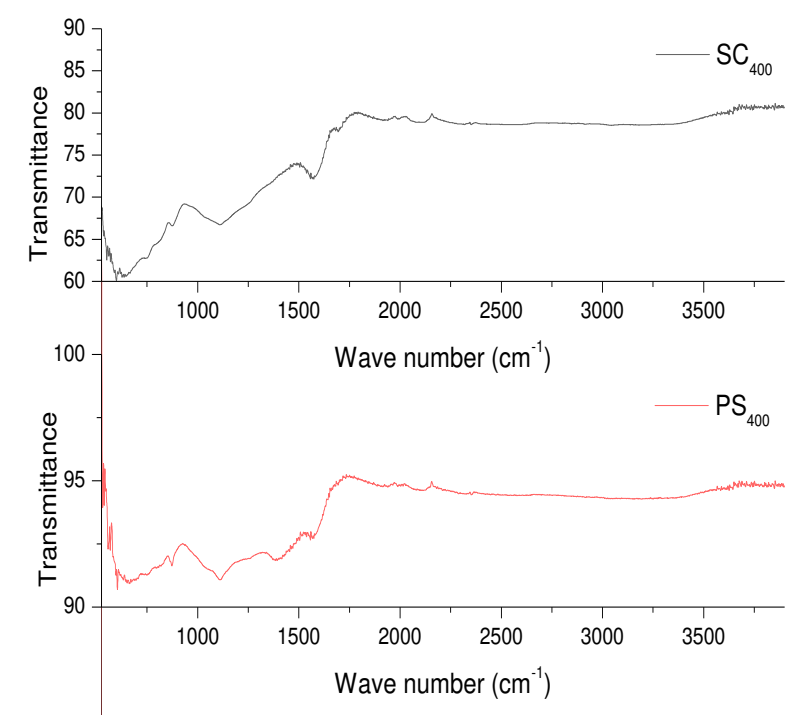

Fig .4 FTIR spectra of biofuels samples

Comparing the spectra of the biomass samples with the biochar samples, we HAVE observed a decrease in the intensity of the C-O-C peaks and the alcohol -OH (1160-1030 cm-1). The biochar spectra HAS showN that pyrolysis at $400^{\circ}$ $\mathrm{C}$ caused degradation of cellulose and hemicellulose. The FTIR spectra of the biomasses HAS ALSO revealed the presence of a broad peak at $3280 \mathrm{~cm}-1$ related to the $\mathrm{OH}$ valency variation of the hydroxyl groups and a peak at 2926 related to the aliphatic $\mathrm{CH}$ elongation vibration in that both peaks have been completely removed from the biochar spectrum. Similarly, the peak corresponding to the variation of the aromatic $\mathrm{C}=\mathrm{C}$ valence $(1620 \mathrm{~cm}-1)$ HAS disappeared from the biochar spectra to decompose into volatile matter during the pyrolysis. There is a lack of peaks between 700 and $900 \mathrm{~cm}-1$ on the biomass spectrum illustrating that the pyrolysis of biomass is a transient process from the aliphatic composition to the aromatic composition [24].

\section{Samples' thermal degradation behavior}

Figure 5 and 6 show the TG and DTG curves of peanut shell, sugar cane and their biochar. The biomass samples' combustion HAS TAKEN place in three main stages. While the first step happens from 20 to $110^{\circ} \mathrm{C}$ corresponding to the loss of water and the evacuation of light molecules, the second step is attributed to the volatile compounds' evacuation generated by the hemicellulose and cellulose' decomposition. For the third stage, it is concerned with the decomposition of lignin and the combustion of coal. Devolatilization has two peaks in that the first one is due to the decomposition of hemicellulose, and the second is caused by the cellulose's decomposition $[5,13]$. Peanut shell and sugar cane Biochar combustion occurs in two main stages. For the first stage, it is between 30 and $130{ }^{\circ} \mathrm{C}$ corresponding to the evacuation of water. As for the second one, it is characterized by a large peak at $505^{\circ} \mathrm{C}$ which is attributed to the combustion of coal. Volatilization peaks do not appear on the DTG curve because most of the volatiles were released during slow pyrolysis. 

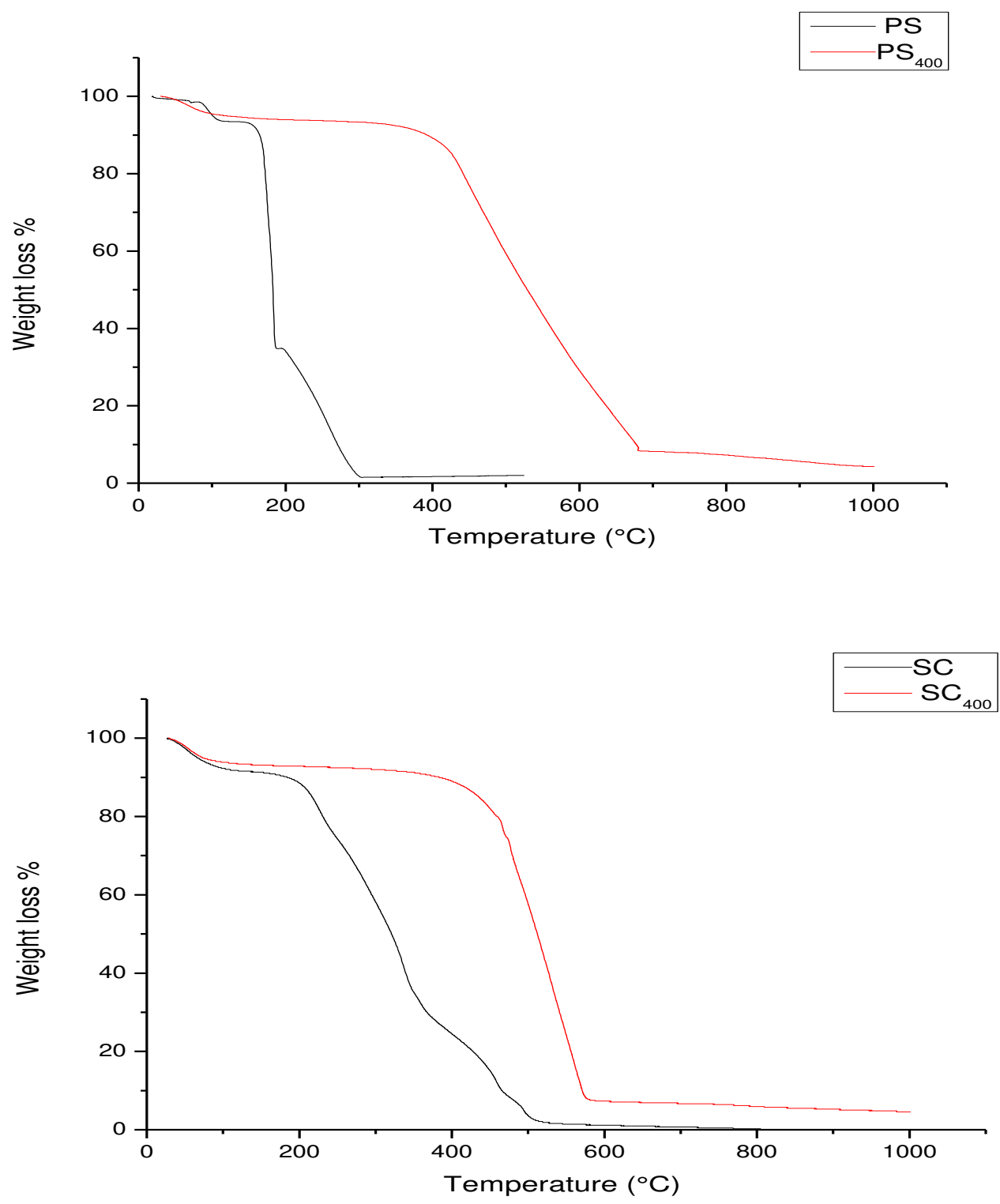

Fig 5 TGA of biomasses and biofuels samples 


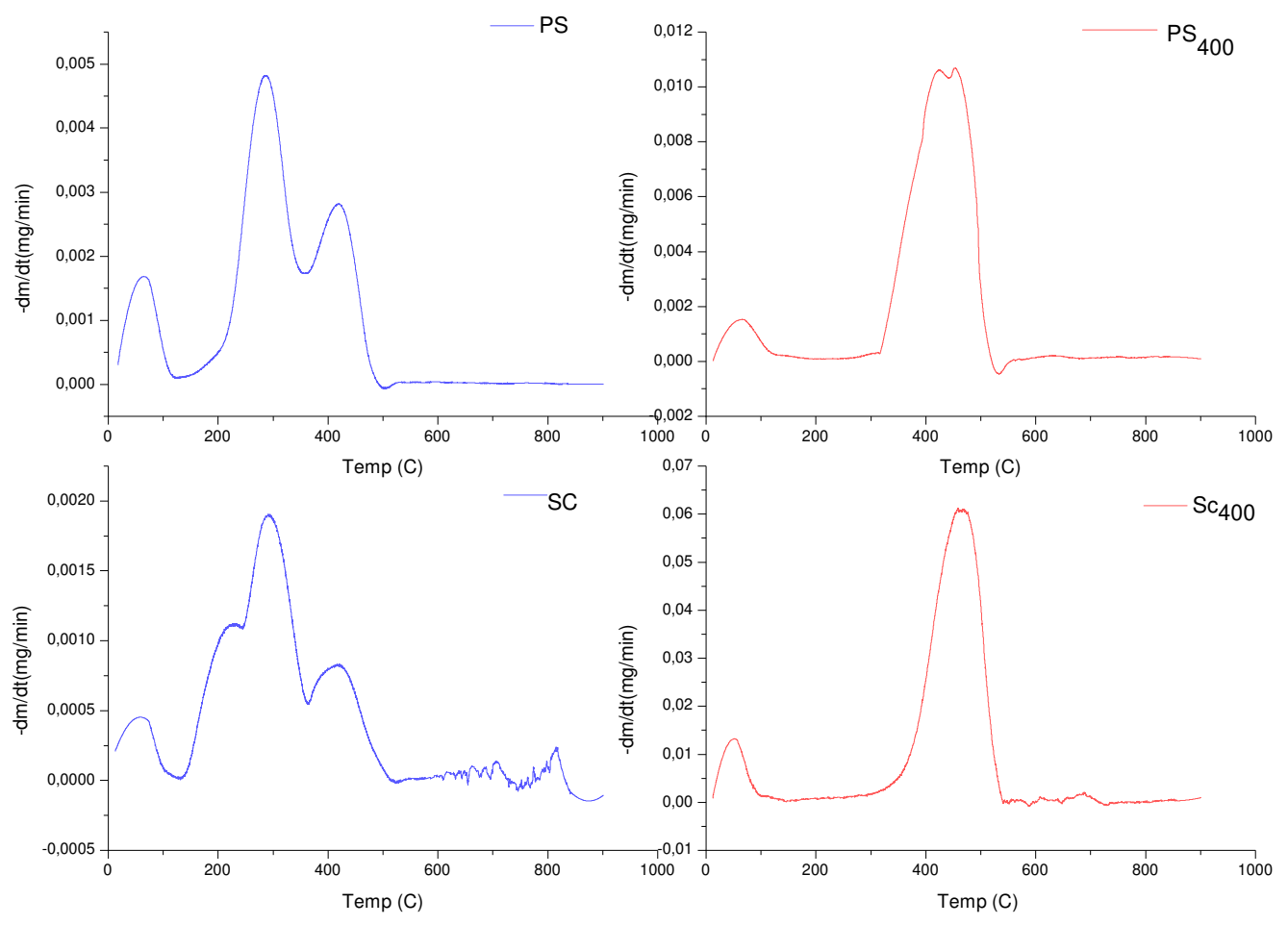

Fig 6 DTG curves of biomasses and biofuels samples

\section{Kinetic behavior}

The activation energy and the frequency factor THAT ARE calculated from the slope of the Coats- Redfern plots are presented in Table (4 and 5). The mass loss kinetics for the samples studied IS determined according to the highest correlation coefficient by the model representing the form of g(a) (Table 3). Based on coefficients correlation value (R2), we HAVE chosen the appropriate models. Indeed, the chosen models present an R2 between 0.89 and 0.99 , which shows that the mechanisms are justified. Table 4 and 5 demonstrate the kinetic parameters (Ea, A) of combustion step of the raw biomass and its derived biochar.

Table 4 Biomasses samples' Kinetic parameters using various reaction models

\begin{tabular}{llllll}
\hline & $\mathrm{T}\left(\mathrm{C}^{\circ}\right)$ & Model & $\mathrm{Ea}(\mathrm{Kj} / \mathrm{mol})$ & $\mathrm{A}\left(\mathrm{min}^{-1}\right)$ & $\mathrm{R} 2$ \\
\hline \multirow{2}{*}{ PS } & $216-361$ & $\mathrm{D} 3$ & 16.45 & 2062779.34 & 0.99 \\
\cline { 2 - 6 } & $361-500$ & $\mathrm{D} 3$ & 9.63 & 52432.62 & 0.95 \\
\hline \multirow{2}{*}{$\mathrm{SC}$} & $172-365$ & F3 & 17.37 & 3148077.45 & 0.98 \\
\cline { 2 - 6 } & $365-500$ & D3 & 10.64 & 82745.62 & 0.99 \\
\hline
\end{tabular}

Table 5 Biofuels samples' Kinetic parameters using various reaction models

\begin{tabular}{llllll}
\hline & $\mathrm{T}\left(\mathrm{C}^{\circ}\right)$ & Model & $\mathrm{Ea}(\mathrm{KJ} / \mathrm{mol})$ & $\mathrm{A}\left(\mathrm{min}^{-1}\right)$ & $\mathrm{R} 2$ \\
\hline $\mathrm{PS}_{400}$ & $308-530$ & $\mathrm{~F} 2$ & 47.22 & 779427226 & 0.89 \\
\hline
\end{tabular}


According to the results shown in table 4, the kinetic parameters characterizing the process of combustion of biomass samples differ from one stage to another and from one sample to another. The combustion of two biomass samples, which occurred in two stages, IS better characterized by a diffusion control mechanism for the first zone of PS , and is better described by the third order chemical reaction model F3 for the first zone of SC. The second zone IS best characterized by a diffusion controlled mechanism for the two samples. This suggests that the diffusion of oxygen to the char particle and of volatiles substances controls the combustion process from ignition to burnout.

By calculating the kinetic parameters using the models that have given the best correlation, peanut shell HAS an apparent activation energy of $16.45 \mathrm{KJ} / \mathrm{mol}$ for the first zone and $9.63 \mathrm{KJ} / \mathrm{mol}$ for the second one, . For sugar cane, it presenteS a value of $17.37 \mathrm{KJ} / \mathrm{mol}$ and $10.64 \mathrm{KJ} / \mathrm{mol}$ respectively for both the first and the second zone. Comparing this kinetic parameters, we HAVE found that the activation energy of the two samples' combustion steps is classified as follows: $\mathrm{E}$ (zone 1 ) >E (zone 2). This means that the char oxidation stage requires less energy to react than that required in volatile matter release stage.

The combustion of Biochar $\mathrm{PS}_{400}$ and $\mathrm{SC}_{400}$ is controlled by reaction kinetics particularly THE second and third order mechanism. The two biochar samples presenteS activation energies of $47.22 \mathrm{~kJ} / \mathrm{mol}$ and $92.86 \mathrm{KJ} / \mathrm{mol}$ respectively for $\mathrm{PS}_{400}$ and $\mathrm{SC}_{400}$. In this respect, this value HAS showN that bio char HAS had a high activation energy than their parent biomasses. In fact, the combustibility is not only affected by activation energy, but it is also influenced by the pre-exponential factor [25]. The same classification of activation energies is obtained for the pre-exponential factors. The biomass HAS a pre-exponential factor of $2062779.34 \mathrm{~min}^{-1}$ and $3148077.45 \mathrm{~min}^{-1}$ for the first zone and 52432.62 $\mathrm{min}^{-1}$ and $82745.62 \mathrm{~min}^{-1}$ for the second zone successively for PS and SC. Whilethe biochar samples presented a preexponential factor of 779427226 min-1 and $2.8710 * 13$ min $^{-1}$ respectively for $\mathrm{PS}_{400}$ and $\mathrm{SC}_{400}$, the pre-exponential factor reflects the number of collisions between activated molecules that is called an effective collision.

The peanut shell's raw biomass and biochar samples are more reactive and have higher flammability in comparison to the raw biomass and biochar samples of sugar cane since they presented little activation energy whose reactions with high activity energy required high temperature and longer reaction time.

\section{Thermodynamic Parameters}

The change in $\Delta \mathrm{H}$ has shown the differences in the energy existing between the activated complex and the reagents agreed with the activation energies [23,22]. If this difference is small, then, the formation of activated complex is favored because of the potential energy barrier inferiorit [26]. The positive $\Delta \mathrm{H}$ has shown that an external source of energy is required to raise the energy level of the reagents. In addition to that, higher values of the enthalpy indicate a less reactive system. Based on table 6 and 7, lower heat energies is required for the biomass ,mainly in the second zone, than for the biocharbon to oxidate the reagents[27]. Thus, the formation of activated complex is more favored in biochar.

The Gibbs free energy $\Delta \mathrm{G}$ reveals an increase in the the total energy approach of the reagents and the formation of the activated complex. This change is due to MODIFICATION OF THE change $\Delta \mathrm{S}$ and a comprehensive evaluation of the heat flow $\Delta \mathrm{H}$ IN WHICH a higher value of $\Delta \mathrm{G}$ represents a lower favorability of reaction. The biomass has a $\Delta \mathrm{G}$ of 86.39 and 88.07 for the first zone and 114.44 and 111.64 for the second zone respectively for PS0 and $\mathrm{SC}_{0}$, while the biochar has values that are higher 156.91 for $\mathrm{PS}_{400}$ and 207.76 for $\mathrm{SC}_{400}$ and has the lowest energy barrier and absorbs the least amount of heat during combustion. According to the obtained results in table 6 and 7 , we notice that $\Delta \mathrm{S}$ has a negative value for ALL samples which indicates that the disorder of the system is decreased and which henceforth confirms the disorder of products resulting resulted from the bond dissociation that is lower than the initial reactants.

Table 6 Thermodynamic parameter estimation of biomasses samples

\begin{tabular}{llllll}
\hline Samples & $\mathrm{Ea}(\mathrm{KJ} / \mathrm{mol})$ & $\mathrm{A}$ & $\mathrm{H}$ & $\mathrm{G}$ & $\mathrm{S}$ \\
\hline PS & 16.45 & 2062779.34 & 14.07 & 86.39 & -0.252
\end{tabular}




\begin{tabular}{llllll} 
& 9.63 & 52432.62 & 7.15 & 114.44 & -0.256 \\
SC & 17.37 & 3148077.45 & 14.96 & 88.07 & -0.252 \\
& 10.64 & 82745.62 & 6.20 & 111.64 & -0.255 \\
\hline
\end{tabular}

Table 7 Thermodynamic parameter estimation of biofuels samples

\begin{tabular}{llllll}
\hline Samples & $\mathrm{Ea}(\mathrm{KJ} / \mathrm{mol})$ & $\mathrm{A}$ & $\mathrm{H}$ & $\mathrm{G}$ & $\mathrm{S}$ \\
\hline $\mathrm{PS}_{400}$ & 47.22 & 779427226 & 43.54 & 156.91 & -0.256 \\
$\mathrm{SC}_{400}$ & 92.68 & $2.8710^{*}$ & 88.83 & 207.76 & -0.256 \\
\hline
\end{tabular}

\section{Conclusion}

The combustion process of peanut shells, sugar cane and their derived biochar have been studied at a heating rate of $5^{\circ} \mathrm{C}$ using a thermogravimetric analysis. The results have shown that:

- $\quad$ The biomass samples (PS and SC) combustion is represented by three steps in which the first step corresponds to the evaporation of moisture, the second to the devolatilization and the third one matches the char formation.

- Biochar combustion occurs in two main stages attributed to the evacuation of water and to the combustion of coal.

- The biomass kinetic study shows that the first zone of combustion is best fitted by diffusion-controlled mechanisms for peanut shell, and by chemical reaction-controlled mechanisms for sugar cane. As far as the second zone is concerned, the two biomass samples are best fitted by diffusion-controlled mechanism.

- The thermodynamic parameters for the reagent active complex formation during combustion are calculated. The high values of $\Delta \mathrm{H}$ and $\Delta \mathrm{G}$ have shown that biochar is less reactive than its original biomass. Also, the $\Delta \mathrm{S}$ values of all biomasses samples and biochar indicates that the disorder of the system has decreased.

The obtained kinetic and thermodynamic parameters are useful in the conception of combustion reactors of peanut shell, sugar cane and their biochar.

\begin{tabular}{|c|c|c|c|}
\hline \multicolumn{4}{|c|}{ Abbreviations } \\
\hline PS & Peanut shell & $\mathbf{d \alpha} / \mathbf{d t}$ & Conversion rate $\% \mathrm{~min}^{-1}$ \\
\hline SC & Sugar cane & & Conversion degree $\%$ \\
\hline $\mathbf{P S}_{400}$ & peanut shell pyrolized at $400{ }^{\circ} \mathrm{C}$ & $\boldsymbol{\beta}$ & Heating rate $\mathrm{K} \cdot \mathrm{min}^{-1}$ \\
\hline $\mathrm{SC}_{400}$ & Sugar cane pyrolized at $400{ }^{\circ} \mathrm{C}$ & $\mathbf{R}^{2}$ & Correlation coefficient \\
\hline TGA & Thermogravimetric analysis & $\mathbf{g}(\alpha)$ & Integral kinetic model \\
\hline DTG & Differential Thermogravimetric & $\mathbf{F}(\alpha)$ & Differential kinetic model \\
\hline FTIR & Fourier transform infrared spectroscopy & $\Delta \mathbf{G}$ & Free Gibbs energy KJ.mol ${ }^{-1}$ \\
\hline $\mathbf{T}$ & Temperature & $\Delta \mathbf{S}$ & Entropy J.mol ${ }^{-1}$ \\
\hline $\mathbf{T}$ & Time & $\Delta \mathbf{H}$ & Enthalpy KJ.mol ${ }^{-1}$ \\
\hline $\mathbf{K}(\mathbf{t})$ & Reaction rate constant & $\mathbf{T m}$ & DTG max peak temperature $\mathrm{K}$ \\
\hline $\mathbf{E} \mathbf{a}$ & Activation energy Kj. $\mathrm{mol}^{-1}$ & $\mathbf{K}_{\mathbf{h}}$ & Boltzmann constant $\left(1.381 * 10^{-23}\right) \mathrm{J} . \mathrm{K}^{-1}$ \\
\hline A & Pré-exponential factor $\mathrm{s}^{-1}$ & $\mathbf{H}_{\mathbf{A}}$ & Plank constant $(6.626 * 10-34$ J.s \\
\hline $\mathbf{R}$ & Universal gaz constant & & \\
\hline
\end{tabular}

\section{Acknowledgements}

Not applicable. 


\section{Authors' contributions}

$\mathrm{HC}$ performed all the experiments and wrote the manuscript. $\mathrm{AMO}$ and $\mathrm{BF}$ contributed to the conception and design of the research; AMA revised the manuscript for important intellectual content.

\section{Funding}

None.

\section{Availability of data and materials}

All datasets used and analyzed in the current study are available from the cor- responding author on reasonable request.

\section{Ethics approval and consent to participate}

Not applicable.

\section{Consent for publication}

All authors agree to publish this manuscript.

\section{Competing interests}

The authors declare that they have no competing interests.

\section{Author details}

1. Engineering Sciences and Trades laboratory, ENSAM, University Moulay Ismail, Meknes, Morocco.

2. Equip Materials and Applied Catalysis Faculty of Science, University Moulay Ismail, Meknes, Morocco

\section{References:}

[1] X. Kai, T. Yang, Y. Huang, Y. Sun, Y. He, and R. Li, "The effect of biomass components on the cocombustion characteristics of biomass with coal," Proc. 2011 2nd Int. Conf. Digit. Manuf. Autom. ICDMA 2011, pp. 1274-1278, 2011.

[2] J. Krasulina, H. Luik, V. Palu, and H. Tamvelius, "Thermochemical co-liquefaction of Estonian kukersite oil shale with peat and pine bark," Oil Shale, vol. 29, no. 3, pp. 222-236, 2012.

[3] P. McKendry, "Energy production from biomass (Part 1): Overview of biomass.," Bioresour. Technol., vol. 83, no. 1, pp. 37-46, 2002.

[4] A. Saraeian, M. W. Nolte, and B. H. Shanks, "Deoxygenation of biomass pyrolysis vapors: Improving clarity on the fate of carbon,” Renew. Sustain. Energy Rev., vol. 104, no. January, pp. 262-280, 2019.

[5] H. L. Chum and R. P. Overend, "Biomass and renewable fuels," Fuel Process. Technol., vol. 71, no. 1-3, pp. 187-195, 2001.

[6] D. Mallick, P. Mahanta, and V. S. Moholkar, "Synergistic Effects in Gasification of Coal/Biomass Blends: Analysis and Review," pp. 473-497, 2018.

[7] M. L. Kubacki, A. B. Ross, J. M. Jones, and A. Williams, "Small-scale co-utilisation of coal and biomass," Fuel, vol. 101, pp. 84-89, 2012.

[8] G. Lopez, M. Olazar, M. Amutio, J. Bilbao, R. Aguado, and M. Artetxe, "Kinetic study of lignocellulosic biomass oxidative pyrolysis," Fuel, vol. 95, pp. 305-311, 2011.

[9] S. Munir, S. S. Daood, W. Nimmo, A. M. Cunliffe, and B. M. Gibbs, "Thermal analysis and devolatilization 
kinetics of cotton stalk, sugar cane bagasse and shea meal under nitrogen and air atmospheres," Bioresour. Technol., vol. 100, no. 3, pp. 1413-1418, 2009.

[10] A. W. Palumbo, C. J. Bartel, J. C. Sorli, and A. W. Weimer, "Characterization of products derived from the high temperature flash pyrolysis of microalgae and rice hulls," Chem. Eng. Sci., vol. 196, no. xxxx, pp. 527$537,2019$.

[11] Z. Hu et al., "Thermogravimetric analysis of co-combustion of biomass and biochar," J. Therm. Anal. Calorim., vol. 112, no. 3, pp. 1475-1479, 2012.

[12] S. G. Sahu, P. Sarkar, N. Chakraborty, and A. K. Adak, "Thermogravimetric assessment of combustion characteristics of blends of a coal with different biomass chars," Fuel Process. Technol., vol. 91, no. 3, pp. 369-378, 2010.

[13] D. López-González, M. Fernandez-Lopez, J. L. Valverde, and L. Sanchez-Silva, "Kinetic analysis and thermal characterization of the microalgae combustion process by thermal analysis coupled to mass spectrometry," Appl. Energy, vol. 114, pp. 227-237, 2014.

[14] J. Riaza et al., "Combustion of single biomass particles in air and in oxy-fuel conditions," Biomass and Bioenergy, vol. 64, pp. 162-174, 2014.

[15] A. Magdziarz and M. Wilk, "Thermogravimetric study of biomass, sewage sludge and coal combustion," Energy Convers. Manag., vol. 75, no. 2013, pp. 425-430, 2013.

[16] S. Li, S. Xu, S. Liu, C. Yang, and Q. Lu, "Fast pyrolysis of biomass in free-fall reactor for hydrogen-rich gas," Fuel Process. Technol., vol. 85, no. 8-10, pp. 1201-1211, 2004.

[17] Y. El may, M. Jeguirim, S. Dorge, G. Trouvé, and R. Said, "Experimental investigation on gaseous emissions from the combustion of date palm residues in laboratory scale furnace," Bioresour. Technol., vol. 131, pp. 94-100, 2013.

[18] Y. El may, M. Jeguirim, S. Dorge, G. Trouvé, and R. Said, "Study on the thermal behavior of different date palm residues: Characterization and devolatilization kinetics under inert and oxidative atmospheres," Energy, vol. 44, no. 1, pp. 702-709, 2012.

[19] A. B. Phadnis and V. V. Deshpande, "Determination of the kinetics and mechanism of a solid state reaction. A simple approach,” Thermochim. Acta, vol. 62, no. 2-3, pp. 361-367, 1983.

[20] M. V Gil, D. Casal, C. Pevida, J. J. Pis, and F. Rubiera, "Bioresource Technology Thermal behaviour and kinetics of coal / biomass blends during co-combustion,” Bioresour. Technol., vol. 101, no. 14, pp. 56015608, 2010.

[21] V. Dhyani and T. Bhaskar, Kinetic Analysis of Biomass Pyrolysis. Elsevier B.V., 2018.

[22] S. H. Kim, "Investigation of Thermodynamic Parameters in the Thermal Decomposition of Plastic Waste Waste Lube Oil Compounds," vol. 44, no. 13, pp. 5313-5317, 2010.

[23] B. Chen, D. Zhou, and L. Zhu, "Transitional adsorption and partition of nonpolar and polar aromatic contaminants by biochars of pine needles with different pyrolytic temperatures," Environ. Sci. Technol., vol. 42, no. 14, pp. 5137-5143, 2008.

[24] M. Keiluweit, P. S. Nico, and M. G. Johnson, "Dynamic Molecular Structure of Plant Biomass-derived Black Carbon(Biochar)- Supporting Information -," vol. 44, no. 4, pp. 1-19, 2010.

[25] Q. Wang, G. Wang, J. Zhang, J. Y. Lee, H. Wang, and C. Wang, "Combustion behaviors and kinetics analysis of coal, biomass and plastic," Thermochim. Acta, vol. 669, no. June, pp. 140-148, 2018.

[26] L. T. Vlaev, V. G. Georgieva, and S. D. Genieva, "Products and kinetics of non-isothermal decomposition of vanadium(IV) oxide compounds," J. Therm. Anal. Calorim., vol. 88, no. 3, pp. 805-812, 2007.

[27] L. Huang et al., "Thermodynamics and kinetics parameters of co-combustion between sewage sludge and water hyacinth in $\mathrm{CO} 2 / \mathrm{O} 2$ atmosphere as biomass to solid biofuel," Bioresour. Technol., vol. 218, pp. 631642, 2016. 


\section{Figures}

展

\section{Figure 1}

The studied biomass species. a. peanut b. bagasse sugar cane

a

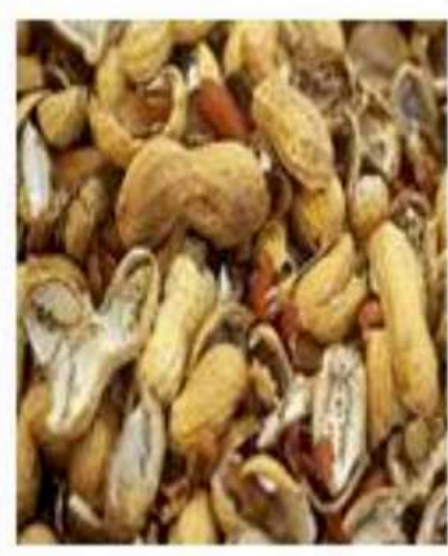

b

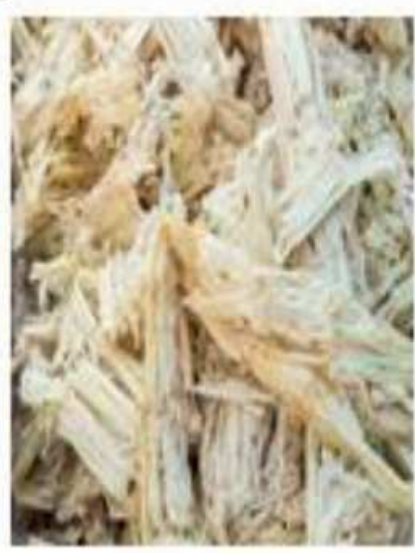

C

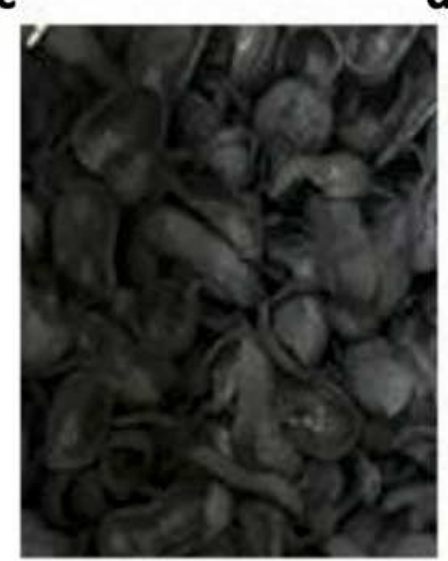

d

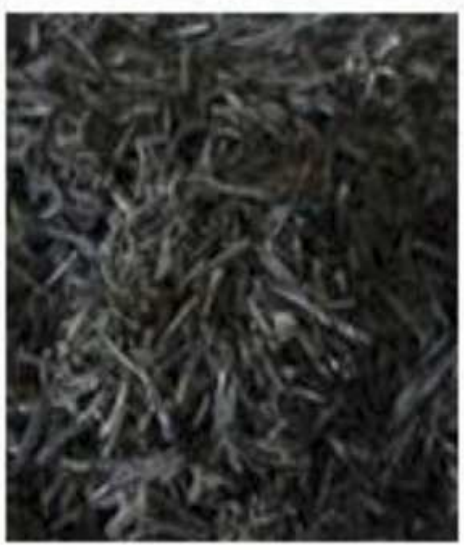

Figure 2

Biomasses and solid biofuel samples. a. Peanut shell (PS) b. sugar cane (PS) c. peanut shell solid biofuel (PS400) d. sugar cane solid biofuel (PS400) 


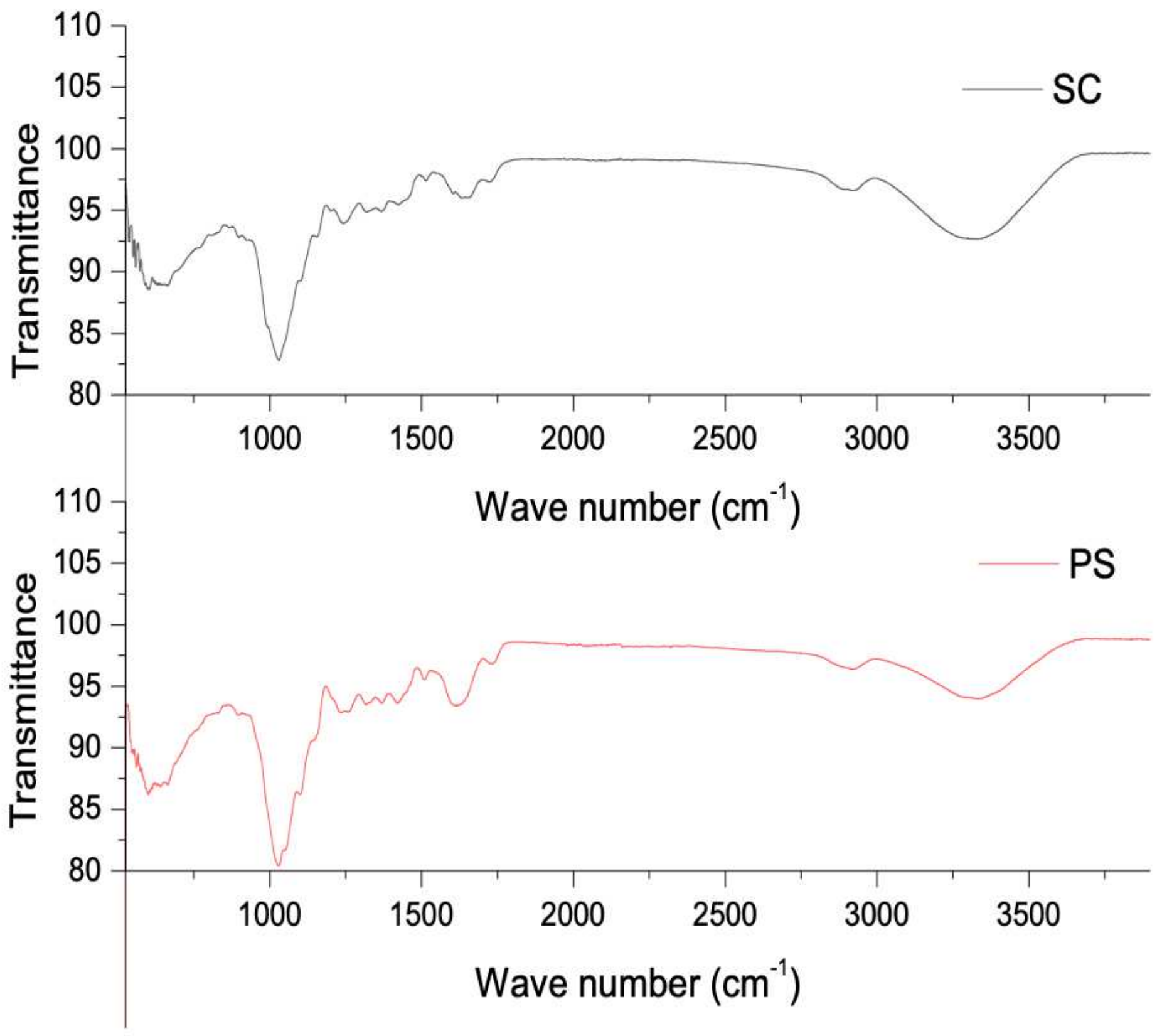

Figure 3

FTIR spectra of biomasses samples 


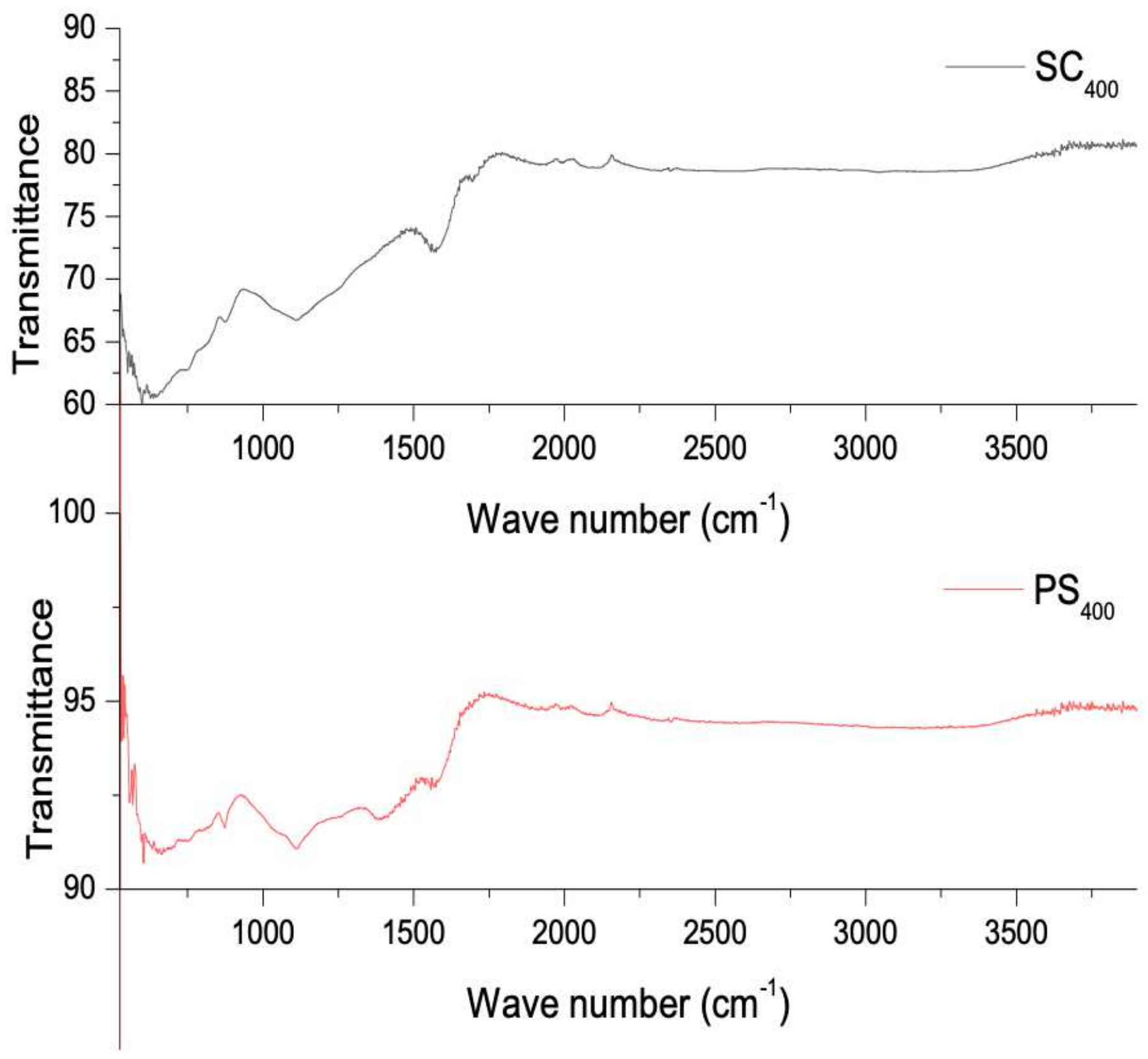

Figure 4

FTIR spectra of biofuels samples 

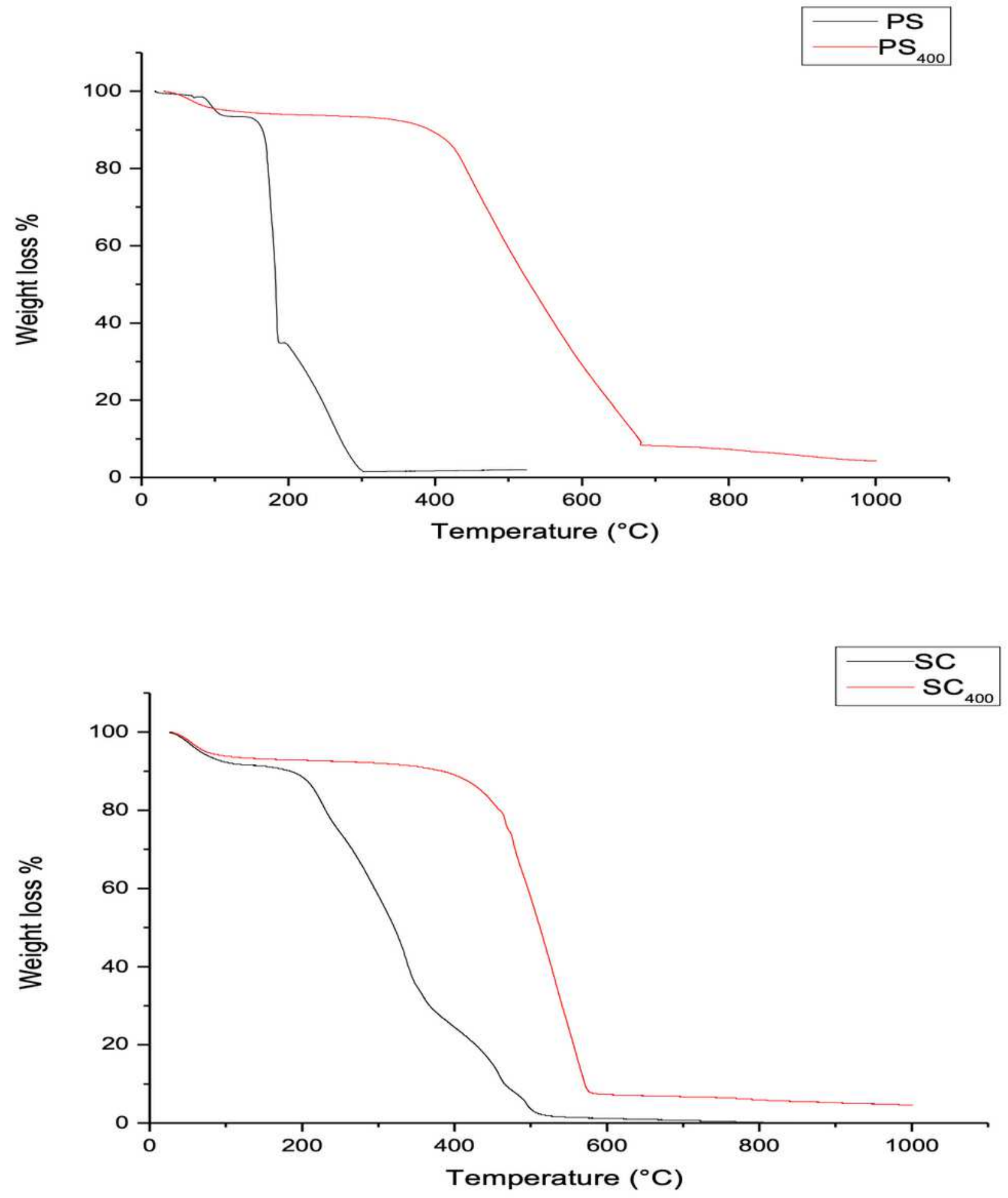

Figure 5

TGA of biomasses and biofuels samples 

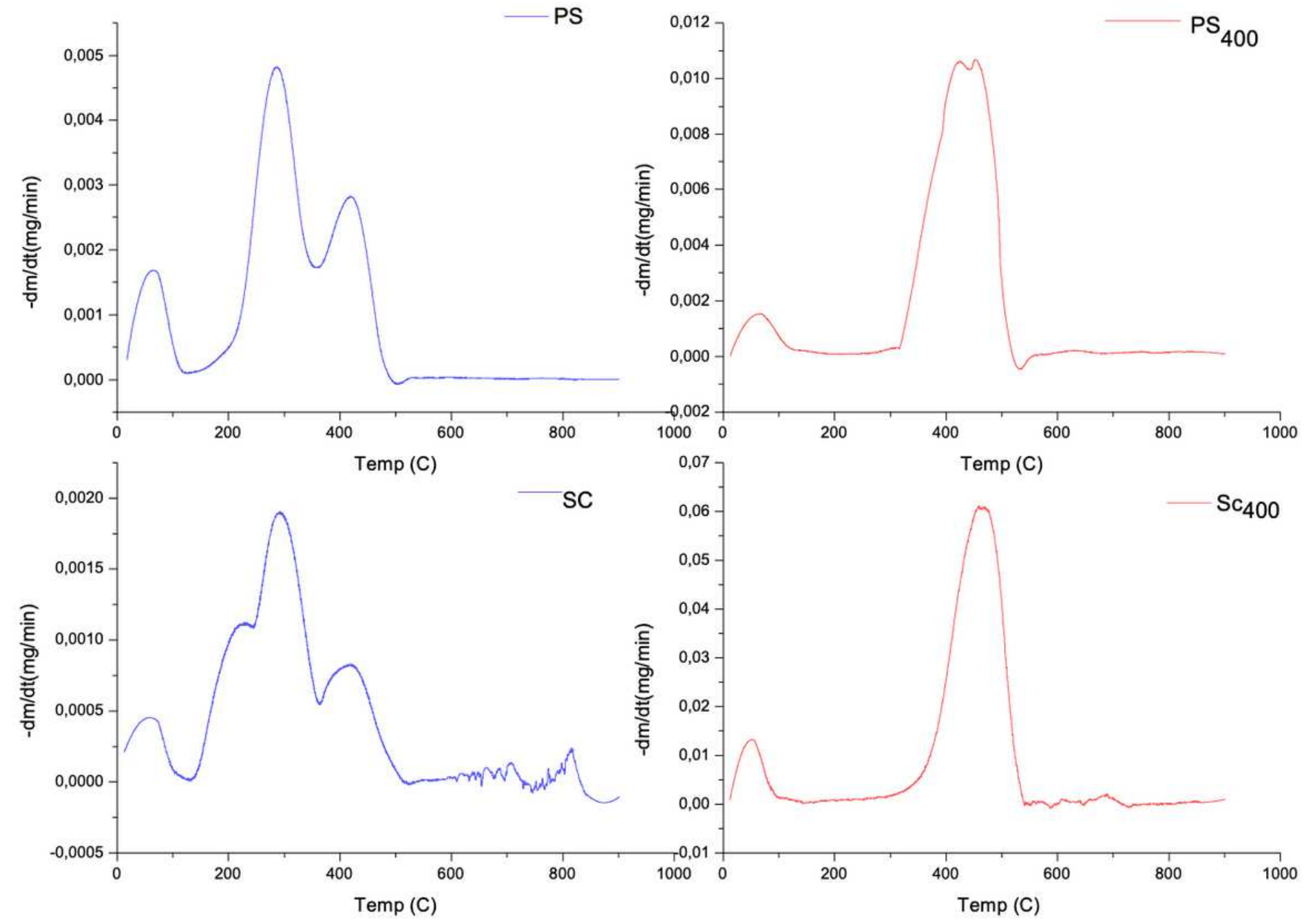

Figure 6

DTG curves of biomasses and biofuels samples 